\title{
Cathodic Reduction of Benzoic Acid in Acidic Solutions*
}

\author{
Isao TANIGUCHI**, Akiko YoshIYAMA** and Taro SEKINE**
}

\begin{abstract}
A controlled-potential electrolysis of benzoic acid was carried out in aqueous and alcoholic $0.5 \mathrm{M}$ $\mathrm{H}_{2} \mathrm{SO}_{4}$ solutions. The results obtained with various cathode materials led to a conclusion that the adsorption of hydrogen and/or benzoic acid onto the electrode surface gave an important influence on the current efficiency for producing benzyl alcohol.

With a lead cathode, the specific adsorption of benzoic acid resulted in a high current efficiency for producing benzyl alcohol by both accelerating the rate of reduction of benzoic acid and inhibiting evolution of hydrogen gas. On the other hand, no remarkable adsorption of benzoic acid was observed on a mercury cathode in the potential region where the reduction of benzoic acid occurred. The reaction of benzoic acid at the lead electrode may be explained in terms of the formation of a protonated benzoic acid molecule as a reacting species, while at a mercury electrode the species was probably a neutral molecule.

The different current efficiencies were obtained for the reduction of benzoic acid to benzaldehyde at various cathodes. However, the reduction of benzaldehyde molecules produced was not affected by the cathode materials.
\end{abstract}

\section{Introduction}

Changes of products and rates of an electrode reaction with different electrode materials have been pointed out by many workers ${ }^{1\}, 2)}$. Upon electroreduction of benzoic acid, (i) cyclohexanecarboxylic acid was obtained at a platinumblack electrode ${ }^{3)}$. However, (ii) benzilic dimer was a main product at a germanium semiconductor electrode ${ }^{4}$. (iii) With metals of high hydrogen overvoltage, benzaldehyde and benzyl alcohol were obtained ${ }^{5)}$ (9). In the last case the following facts are reported : At a mercury electrode $^{5), 6)}$ benzyl alcohol and benzaldehyde are formed in acidic and neutral solutions, respectively. At a lead cathode benzyl alcohol is formed with a high current efficiency in an acidic solution ${ }^{37,7), 8)}$, whereas no appreciable reduction of benzoic acid is observed in a neutral solution ${ }^{6}$. Swann and Luker $^{9}$ reported that only lead and cadmium were effective cathodes for the reduction in $30 \% \mathrm{H}_{2} \mathrm{SO}_{4}$ solution. However, the reason why lead is an excellent cathode

* A part of this work was presented at the $33 \mathrm{rd} \mathrm{Me}-$ eting of the Chemical Society of Japan, Fukuoka, October 1975.

** Department of Electronic Chemistry, Tokyo Institute of Technology, Graduate School at Nagatsuta (2-12-1 Ookayama, Meguro-ku, Tokyo) for producing benzyl alcohol has not been clear.

In the present work, the electroreduction of benzoic acid to benzyl alcohol in acidic solutions was carried out at different electrode materials and potentials, and the adsorption characteristics of benzoic acid onto the electrode surface was investigated.

\section{Experimental}

\subsection{Electrolysis and reagents}

The electrolysis was carried out by controlling the cathode potential under vigorous agitation. The potential was measured with a potentiometer (TOA PM 18 C) during each run. A saturated calomel electrode (S.C.E.) was used as a reference electrode. The potentials given in this paper are referred to this electrode.

The electrolytic cell was an H-type cell which was divided into cathode and anode compartments by a fine glass frit. The anode was a platinum sheet $\left(4 \mathrm{~cm}^{2}\right)$ and the anolyte was $35 \mathrm{ml}$ of $0.5 \mathrm{M} \mathrm{H}_{2} \mathrm{SO}_{4}$. For the cathode, several metals $(\mathrm{Pb}, \mathrm{Cd}, \mathrm{Hg}, \mathrm{Zn}, \mathrm{Al}, \mathrm{Ti}, \mathrm{Cu}, \mathrm{Fe}$ and $\mathrm{Ni}$ ) with $99.9 \%$ purity were used. Mercuryplated electrodes were prepared by the electrolytic plating of mercury on either lead or copper in $\mathrm{Hg}\left(\mathrm{NO}_{3}\right)_{2}$ solution $^{10)}$. The surface area of 
all cathodes used was $10 \mathrm{~cm}^{2}$ except for the mercury pool $\left(20 \mathrm{~cm}^{2}\right)$. The volume of the catholyte was $100 \mathrm{ml}$.

Oxygen evolved at the anode was allowed to flow out through a pressure regulator to maintain the level of the catholyte constant during the experiments. Diffusion of benzoic acid and benzyl alcohol through the frit was negligibly small. The volume of hydrogen gas was measured by a gas buret.

Among the reagents used, benzoic acid(reagent grade GR) was recrystallized twice from water and others (GR) were used without further purification.

\subsection{Analytical}

The concentration of benzoic acid was determined with a UV spectrometer (Hitachi Model 624). The absorbance of the electrolyte at $230.5 \mathrm{~nm}$ was measured before and after each run. After a run, sodium chloride crystals were added to the catholyte and then benzyl alcohol and benzaldehyde were extracted with three portions of $30 \mathrm{ml}$ ether. The extract was analyzed by a gas chromatograph (Yanaco G-1800) by using a column of polyethylene glycol $20 \mathrm{M}$. No product other than benzaldehyde and benzyl alcohol was observed*1. The error of the analysis was within $\pm 3 \%$.

\subsection{Differential capacity measurement}

The differential capacity of the electrode at the electrode-electrolyte interface was measured by a usual a.c. impedance bridge $\operatorname{method}^{11}$. The working electrodes used were a micro-lead electrode $\left(0.07 \mathrm{~cm}^{2}\right)$ and a dropping mercury electrode (D.M.E.) with a drop-life of 11-13 s. The test solution was deaerated by bubbling purified nitrogen which had been pre-equilibrated with vapor of a solution having the same composition as that of the test solution.

\section{Results and Discussion}

The results of controlled-potential electrolysis with a lead cathode are shown in Fig. 1. The reduction of benzoic acid to benzyl alcohol and the hydrogen evolution reaction (h.e.r.) occurred simultaneously. Although the partial current

*1 The benzoic acid solution with ethyl alcohol was always freshly prepared to prevent the formation of ethyl benzoate in a stock solution.

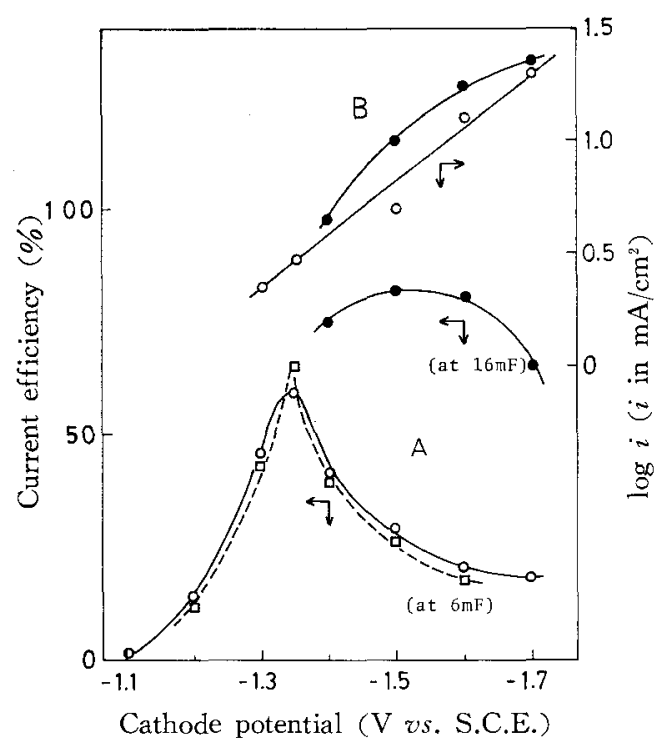

Fig. 1 Influence of cathode potential on the current efficiency and the current for reduction of benzoic acid on a lead electrode at $25^{\circ} \mathrm{C}$

$$
\begin{aligned}
& : 0.5 \mathrm{M} \mathrm{H}_{2} \mathrm{SO}_{4}+10 \mathrm{mM} \text { benzoic acid } \\
\square & : 1 \mathrm{M} \mathrm{HClO}_{4}+10 \mathrm{mM} \text { benzoic acid } \\
: & 0.5 \mathrm{M} \mathrm{H}_{2} \mathrm{SO}_{4}+30 \% \mathrm{v} / \mathrm{v} \text { ethyl alcohol } \\
& +100 \mathrm{mM} \text { benzoic acid }
\end{aligned}
$$

consumed by the reduction of benzoic acid*2 increased with the cathode potential (Fig. 1 B), the current for the h.e.r. increased more rapidly than that for the reduction of benzoic acid with decreasing cathode potential, and therefore, a maximum current efficiency for producing benzyl alcohol was found at $-1.35 \mathrm{~V}$ in the aqueous solution and $-1.50 \mathrm{~V}$ in the alcoholic solution (Fig. 1 A). Benzoic acid was not reduced at more noble potentials than $-1.20 \mathrm{~V}$ where the h.e.r. occurred.

To examine the role of sulfate anion, the electrolysis was also carried out in $\mathrm{HClO}_{4}$ solution. No remarkable difference in current efficiency was observed between solutions con-

*2 To compare the currents at a constant concentration of benzoic acid, the current at the initial concentration of benzoic acid was estimated as follows: The change of reduction current, $i$, of benzoic acid with time was first calculated by using the equation $i=i_{\mathrm{t}}$ $-i_{\mathrm{h}}$, where $i_{\mathrm{t}}$ is the total current observed and $i_{\mathrm{h}}$ is the current consumed by the h.e.r. calculated from the volume of hydrogen evolved, and then the calculated $i^{\prime} s$ at various durations of electrolysis were extrapolated toward time zero. 
Table 1 Effect of cathode material on the current efficiency of the reduction of benzoic acid

$\mathrm{A}$ : Aqueous $0.5 \mathrm{M} \mathrm{H}_{2} \mathrm{SO}_{4}$ solution containing $10 \mathrm{mM}$ benzoic acid, at $-1.4 \mathrm{~V}$, at $30^{\circ} \mathrm{C}$

\begin{tabular}{l|c|c|c|c|c|c|c|c}
\hline \multicolumn{1}{c|}{ Cathode } & $\mathrm{Pb}$ & $\mathrm{Cd}$ & $\mathrm{Hg}$ pool & $\mathrm{Hg}-\mathrm{Cu}$ & $\mathrm{Hg}-\mathrm{Pb}$ & $\mathrm{Zn}$ & $\mathrm{Al}$ & $\mathrm{Ti}$ \\
\hline Current consumed $(\mathrm{mF})$ & 5.17 & 5.66 & 5.40 & 5.92 & 6.29 & 5.19 & 5.40 & 5.30 \\
\hline Current efficiency (\%) & & & & & & & & \\
Benzyl alcohol & 51.5 & 25.2 & 18.5 & 3.8 & 6.4 & 2.7 & 0.6 & 0.9 \\
Benzaldehyde & ++ & ++ & ++ & ++ & + & + & + & + \\
Hydrogen & 44.5 & 76.3 & 80.0 & 96.0 & 93.4 & 100 & 100 & 100 \\
\hline
\end{tabular}

$++:<0.1 \mathrm{mM}(<0.4 \%$ in current efficiency), + : Trace

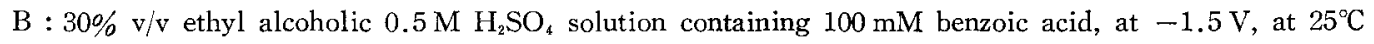

\begin{tabular}{l|c|c|c|c|c|c|c|c}
\hline \multicolumn{1}{c}{ Cathode } & $\mathrm{Pb}$ & $\mathrm{Cd}$ & $\mathrm{Hg}$ pool & $\mathrm{Hg}-\mathrm{Cu}$ & $\mathrm{Hg}-\mathrm{Pb}$ & $\mathrm{Zn}$ & $\mathrm{Al}$ & $\mathrm{Ti}$ \\
\hline Current consumed $(\mathrm{mF})$ & 16.0 & 15.1 & 15.5 & 14.9 & 15.3 & 15.0 & 15.5 & 15.7 \\
\hline Current efficiency $(\%)$ & & & & & & & & \\
Benzyl alcohol & 83.9 & 44.1 & 40.2 & 1.2 & 1.9 & 2.2 & 0.1 & 0.1 \\
Benzaldehyde & ++ & ++ & ++ & + & + & + & + & + \\
Hydrogen & 17.0 & 58.1 & 62.8 & 100 & 98.0 & 100 & 100 & 100 \\
\hline
\end{tabular}

$++:<0.2 \mathrm{mM}(<0.3 \%$ in current efficiency $),+$ : Trace

taining sulfuric acid and perchloric acid as shown in Fig. 1, i.e., sulfate anion had no influence on the reduction.

Metals may be divided into two groups according to the degree of hydrogen overvoltage $^{12,13)}$. One is called the sp-metal and the other is the $d$-metal. In the former the rate determining step in the h.e.r. is the discharge step of hydrogen ions at the surface of the metal. In the latter, recombination of hydrogen atoms or the ion-plus-atom reaction in the h.e.r. is the rate determining. Since the degree of hydrogen overvoltage is closely related to the reaction mechanism of hydrogen evolution at an electrode surface, the above classification of metals may be useful to characterize electrode materials at which the h.e.r. occurs. It has been known that the different reactivities of an organic compound at various electrode materials were closely related to the nature of metals which affected to the mechanism of the h.e.r. To examine whether or not the classification of metals for the mechanisms of the h.e.r. is useful for characterizing the current efficiency for producing benzyl alcohol, the electrolysis was carried out with various cathode materials. The current efficiencies found are listed in Table 1. Besides the metals listed in Table 1, iron, nickel and copper were also studied, but no reduction of benzoic acid was observed at these cathodes. Swann and Luker" ${ }^{9}$ pointed out that no reduction of benzoic acid occurred at mercury in $30 \%$ $\mathrm{H}_{2} \mathrm{SO}_{4}$ with $55 \% \mathrm{v} / \mathrm{v}$ ethyl alcohol at $0.05 \mathrm{~A} /$ $\mathrm{cm}^{2}$. In the present study, however, the reduction occurred at the mercury pool, while the reduction of benzoic acid at the mercury-plated cathodes $^{* 3}$ was small. The different reactivities showed that the mercury-plated cathodes behaved in somewhat different way from a mercury electrode for the reduction of benzoic acid, even though the surface of the mercury-plated cathode was covered with an excess of liquid mercury.

If we take into consideration the electrocatalytic nature of the electrode surface, the results in Table 1 can be explained as follows: The surface of a d-metal was covered with strongly adsorbed hydrogen atoms, and adsorption of benzoic acid onto the electrode surface may be difficult. Thus the reduction of benzoic acid did not occur except for the hydrogenation of a benzene ring at a platinum-black surface ${ }^{3)}$. Aluminium (this is conventionally classified into

\footnotetext{
*8 Tow mercury-plated cathodes of different base metals were used to examine if the base metals had any influence on the results.
} 
a d-metal even though the metal has no d-electrons) and titanium belong to this category. At the surface of an sp-metal of a relatively low hydrogen overvoltage such as zinc or tin, hydrogen molecules may cover the surface of the metal because the reduction of benzoic acid begins at a rather negative potential. No appreciable reduction of benzoic acid was observed on these metals. On the other hand, the adsorption of hydrogen atoms was too weak to be detected on an sp-metal of a high hydrogen overvoltage because the r.d.s. was the discharge step in the h.e.r. Under this surface condition the reduction of benzoic acid occurred. Lead, cadmium and mercury pool electrodes are classified into this category.

The results described above show that the adsorption of hydrogen and benzoic acid onto the electrode surface gives an important influ-

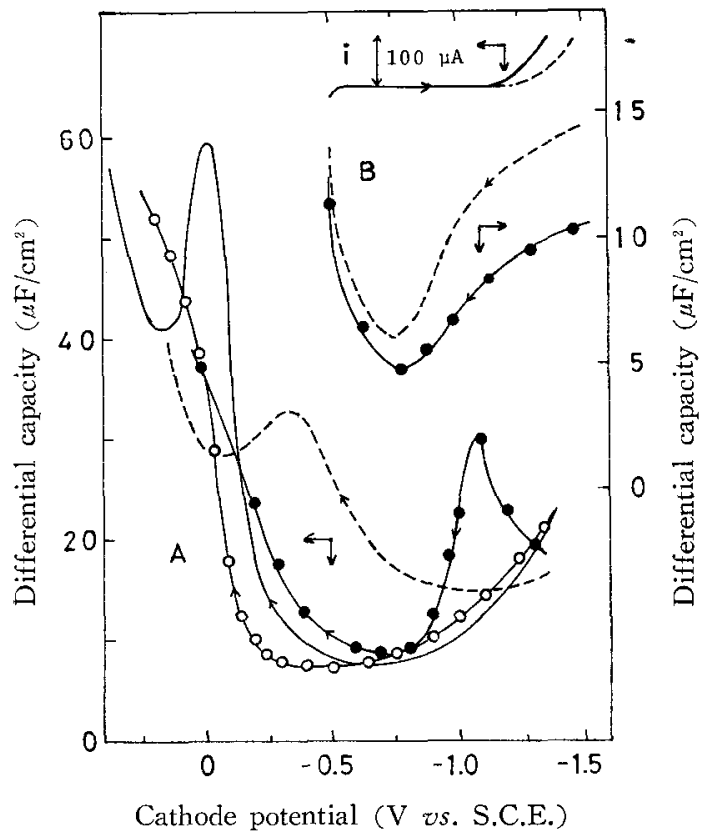

Fig. 2 Differential capacity $v s$. potential curves and polarization curves

A : At a mercury electrode, at $25^{\circ} \mathrm{C}$

$\mathrm{B}:$ At a lead electrode, at $30^{\circ} \mathrm{C}$

...... : $0.5 \mathrm{M} \mathrm{H}_{2} \mathrm{SO}_{4}$

- $: 0.5 \mathrm{M} \mathrm{H}_{2} \mathrm{SO}_{4}+30 \%$ v/v ethyl alcohol

-- : $0.5 \mathrm{M} \mathrm{H}_{2} \mathrm{SO}_{4}+10 \mathrm{mM}$ benzoic acid

-O-: $0.5 \mathrm{M} \mathrm{H}_{2} \mathrm{SO}_{4}+30 \% \mathrm{v} / \mathrm{v}$ ethyl alcohol $+50 \mathrm{mM}$ benzoic acid

The direction of potential sweep is shown by arrows on the lines ence on the current efficiency for producing benzyl alcohol. This consideration was supported by the following result: At a lead cathode, in the aqueous benzoic acid solution containing a tetraethylammonium salt, the current for the reduction of benzoic acid at $-2.0 \mathrm{~V}$ was only $1 / 20$ of the current in the solution without the tetraethylammonium salt at $-1.4 \mathrm{~V}, i . e$, the adsorption of the tetraethylammonium cation prevented the adsorption of benzoic acid. Thus, the adsorption of benzoic acid onto mercury and lead electrodes was directly examined by capacitance measurements. Typical results are shown in Fig. 2, and they may be summerized as follows:

Mercury: The decrease of the capacity due to the addition of benzoic acid was observed in the aqueous $0.5 \mathrm{M} \mathrm{H}_{2} \mathrm{SO}_{4}$ solution, the fact indicating adsorption of benzoic acid. On the capacity curve, however, an adsorption-desorption peak ${ }^{* 4}$ was observed at -1.1 to $-1.2 \mathrm{~V}$ (-) in Fig. 2A), and this indicates that benzoic acid is not adsorbed on the electrode surface in the potential region where the reduction occurs. Two capacity-potential curves measured in the alcoholic $0.5 \mathrm{M} \mathrm{H}_{2} \mathrm{SO}_{4}$ solution with $(-)$ and without $\left(-\mathrm{O}^{-}\right)$benzoic acid did not show any appreciable difference (Fig. $2 \mathrm{~A}$ ), and this fact indicates that the benzoic acid is unable to adsorb by displacing adsorbed ethyl alcohol. It is thus concluded that the adsorption of benzoic acid on mercury gives no marked effect on the reduction of benzoic acid in both aqueous and alcoholic solutions.

Lead: The capacity was decreased by the addition of benzoic acid at a negatively charged surface (Fig. $2 \mathrm{~B}$ ), and this fact suggests that benzoic acid is adsorbed on lead at potentials where the reduction occurs. As shown in Fig. $2 \mathrm{~B}$, at more negative potentials than $-1.2 \mathrm{~V}$ the double layer capacity was unable to be measured directly by an a.c. impedance bridge method because of the faradaic current. Thus, in order to examine the adsorption of benzoic acid at these potentials, the following method was employed. The apparent coverage of benzoic acid $(\theta)$ was determined by comparing the currents for hydrogen evolution with and with-

\footnotetext{
*4 This peak was confirmed to be an adsorption-desorption peak by the measurements of interfacial tension of mercury.
} 


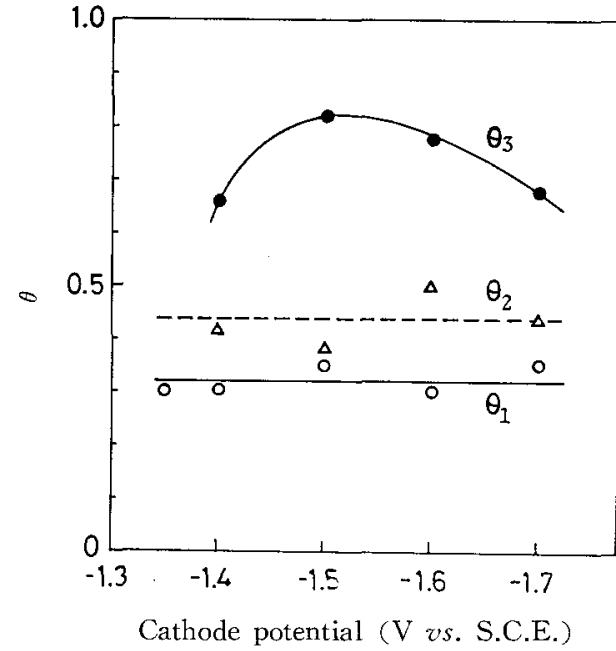

Fig. 3 Coverage $(\theta) v s$. potential curves

$$
\begin{aligned}
& 0: \theta_{1}=1-\left(i_{1} / i_{0}\right), \quad \triangle: \theta_{2}=1-\left(i_{2} / i_{0}\right) \\
& : \theta_{3}=1-\left(i_{3} / i_{2}\right)
\end{aligned}
$$

where $i_{1}$ and $i_{0}$ are the currents for hydrogen evolution in $0.5 \mathrm{M} \mathrm{H}_{2} \mathrm{SO}_{4}$ with and without 10 $\mathrm{mM}$ benzoic acid. $i_{3}$ and $i_{2}$ are the currents for hydrogen evolution in $0.5 \mathrm{M} \mathrm{H}_{2} \mathrm{SO}_{4}+30 \% \mathrm{v} / \mathrm{v}$ ethyl alcohol with and without $100 \mathrm{mM}$ benzoic acid

out benzoic acid at the same potential according to the equation $(1)^{* 5}$;

$$
\theta=1-\left(i / i_{0}\right)
$$

where $i$ and $i_{0}$ are the currents ${ }^{* 6}$ in the presence and absence of benzoic acid at a given potential. The results obtained are shown in Fig. 3. The coverage of benzoic acid was about 0.3 which was independent of potential in the aqueous solution, while in the alcoholic solution the coverage varied with potential and a maximum was observed at $-1.5 \mathrm{~V}$. Thus, the specific adsorption of benzoic acid was evident, even though the inaccuracy of the calculated $\theta$ values was taken into account.

A reaction scheme in which the reduction of benzoic acid to benzyl alcohol proceeded via. benzaldehyde was proposed by Holleck and Marsen ${ }^{16)}$. This proposal was supported by the result obtained in the present work, i.e., a trace amount of benzaldehyde was detected in most electrolytes. Since benzaldehyde is more

*5 This method was widely used ${ }^{(4)}$, but in some cases a deviation of over $10 \%$ was observed when $\theta>0.5^{15}$.

* The value of $i$ was calculated from the volume of hydrogen evolved. easily reduced than benzoic acid, we considered that the step of the effective electrocatalysis might be the reduction process of benzoic acid but not benzaldehyde. In order to give experimental supports for this consideration, we examined the reduction of benzaldehyde under similar conditions. The results obtained are summarized in Table 2. The current efficiencies. with different metals were nearly the same and no appreciable difference between mercury pool and mercury-plated lead electrodes was observed. These results indicate that the adsorption of benzaldehyde is not important for the reduction of benzaldehyde under the present experimental conditions. The partial current consumed by the reduction of benzaldehyde*r increased almost linearly with the degree of polarization from the zero charge potentials of the metals $^{\left.17,{ }^{19}\right)}$ as shown in Fig. 4. The linear dependence of $\log i$ on $E-E_{\mathrm{p} z c}$ may indicate the so-called $\psi$ effect ${ }^{19}$ ) (an effect of double layer structure on the rate of reactions without specific adsorption) if we assume the protonated benzaldehyde as a reacting species in an acidic solution $^{209}$.

In the case of the reduction of benzoic acid at the lead cathode under the present experimental conditions, the charge transfer reaction is probably the r.d.s., since the partial current for the reduction of benzoic acid increased with decrcasing cathode potential, whereas the current did not increase linearly with the concentration of benzoic acid (Fig. 1). Thus, only adsorbed molecules seem to take part in the reaction. Then the relationship between the coverage of benzoic acid and the partial current consumed by the reduction of benzoic acid was tested. In the alcoholic solution, since a part of the surface is covered by ethyl alcohol and the apparent coverage of benzoic acid, $\theta_{3}$, is calculated from the surface area partially covered with ethyl alcohol (see the definition of $\theta_{3}$ in Fig. 3 ), the true coverage of benzoic acid molecules with respect to the total surface may be given by Eq. (2)*8;

$$
\theta_{\mathrm{t}}=\theta_{3}\left(1-\theta_{2}\right)
$$

\footnotetext{
*7 The current was calculated in the same manner as. that for benzoic acid.

* It is assumed that no reduction occurs at the electrode surface covered with ethyl alcohol molecules.
} 
Table 2 Effect of cathode material on the current efficiency of the reduction of benzaldehyde in aqueous $0.5 \mathrm{M} \mathrm{H}_{2} \mathrm{SO}_{4}$ containing $10 \mathrm{mM}$ benzaldehyde at $30^{\circ} \mathrm{C}$

\begin{tabular}{c|c|c|c|c|c}
\hline \hline \multirow{2}{*}{ Cathode } & $\begin{array}{c}\text { Cathode potential } \\
\text { (V vs. S.C.E.) }\end{array}$ & $\begin{array}{c}\text { Current consumed } \\
(\mathrm{mF})\end{array}$ & \multicolumn{3}{|c}{ Current efficiency (\%) } \\
\cline { 3 - 6 } & -1.0 & 1.31 & $<5$ & $>95$ & 0 \\
\hline $\mathrm{Pb}$ & -1.4 & 5.50 & -1.0 & - & 68.4 \\
$\mathrm{Cd}$ & -1.0 & 0.99 & -100 & 0 \\
& -1.4 & 4.78 & -10.7 & -100 & 69.6 \\
$\mathrm{Hg}$ & -1.0 & 0.79 & 35.2 & - & 62.4 \\
$\mathrm{Hg}-\mathrm{Pb}$ & -1.4 & 5.34 & 29.8 & - & 70.2 \\
\hline
\end{tabular}

* The formation of hydrobenzoin was confirmed. The current efficiency was estimated from that of benzyl alcohol.

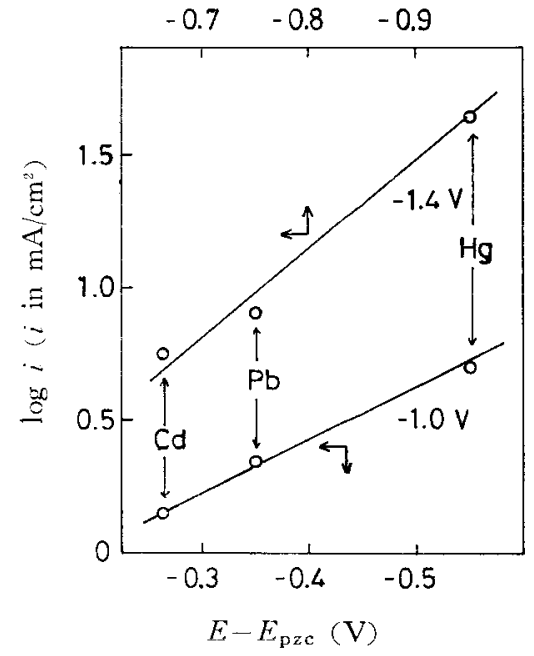

Fig. 4 Relationship between polarization from the zero charge potential and the current for reduction of benzaldehyde

The adopted zero charge potentials were; -0.45 $(\mathrm{Hg}),-0.65(\mathrm{~Pb}),-0.77(\mathrm{Cd}) \mathrm{V}$ vs. S.C.E.

where $\theta_{3}$ and $\theta_{2}$ are apparent coverages of benzoic acid and ethyl alcohol in the alcoholic solution calculated from the equations described in Fig. 3. The $\theta_{2}$ value was estimated as 0.44 (Fig. 3). The ratio of the partial currents for the reduction of benzoic acid in the alcoholic and the aqueous solutions, $i_{\mathrm{a}} / i_{\mathrm{b}}$, was in fairly good agreement with that of the coverages of benzoic acid, $\theta_{\mathrm{t}} / \theta_{\mathrm{l}}$, as shown in Fig. 5. The agreement indicates that the rate of reduction of benzoic acid is proportional to the coverage as represented by the following equation;

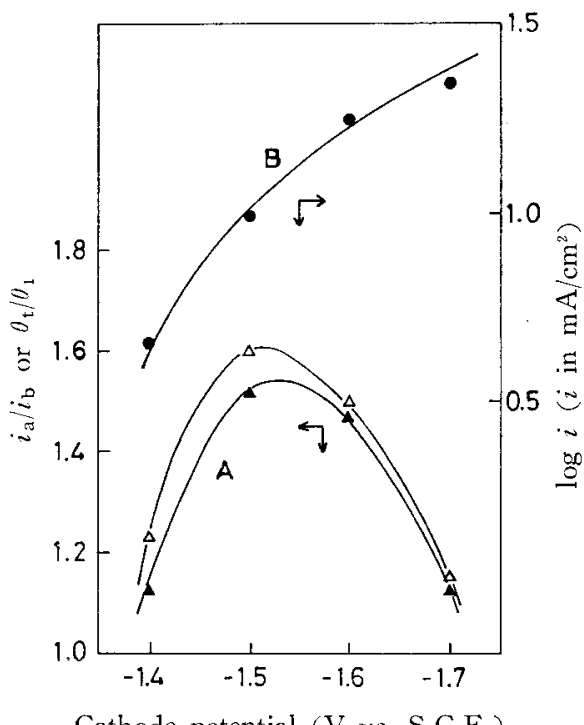

Cathode potential ( $\mathrm{V}$ vs. S.C.E.)

Fig. 5 Relationship between the coverage of benzoic acid and the reduction current as a function of cathode potential

A : $\boldsymbol{A}$; The ratio of the currents for reduction of benzoic acid, $i_{\mathrm{a}} / i_{\mathrm{b}}$

$\triangle$; The ratio of the coverages of benzoic acid, $\theta \mathbf{t} / \theta_{1}$

B : — ; Calculated current-voltage curve by Eq. 3 in the alcoholic solution by assuming that the reaction parameters are the same as those in the aqueous solution

- Experimental results in the alcoholic solution

$i=k_{1} \theta \exp (\alpha n F E / R T)$

where $k_{1}$ is the rate constant and the other symbols have usual significance. The currents calculated by this equation by knowing $\theta$ were 
in good agreement with the observed results (Fig. 5), and the suggestion proposed previously that only adsorbed benzoic acid molecules were reduced was thus supported. The adsorption of benzoic acid resulted in the observed high current efficiency for producing benzyl alcohol, because the reduction rate of benzoic acid increased with increasing $\theta$ and the h.e.r. was partially inhibited. At the mercury electrode, the current efficiency for producing benzyl alcohol, which was lower than that at the lead electrode, was caused by the absence of the adsorption of benzoic acid.

The cause of the specific adsorption of benzoic acid may be considered as follows : Since no reduction of benzoic acid was observed in a weakly acid or neutral solution ${ }^{6, * 9}$ and the adsorption of benzoic acid was found at rather negative potentials as demonstrated above, the electroactive species would be a protonated benzoic acid molecule at a lead cathode, whereas the species would be a neutral molecule at a mercury electrode because the reduction of benzoic acid occurred in a neutral solution ${ }^{57,5)}$ but not in a strong acid solution". Hosoya and Nagakura $^{21}$ showed that the structure of a protonated benzoic acid was given as (I).

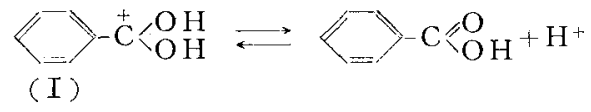

The knowledge of the strength of the bond energy between metal and hydrogen atom ( $\mathrm{Hg}$ $<<\mathrm{Cd}<\mathrm{Pb})^{22,23)}$ and the structure ( I ) led to a conclusion that the protonated benzoic acid would have a strong interaction with a lead electrode, while a interaction was weak with a mercury electrode, by assuming the contact of carboxylic group with an electrode surface. In this case the cause of specific adsorption of benzoic acid on a lead cathode can be explained, and the different reactivities of benzoic acid between mercury and lead electrodes were explained in terms of the difference of the reacting species.

\section{Conclusion}

* No appreciable reduction of benzoic acid was also observed in sodium sulfate solution containing a borate buffer ( $\mathrm{pH} 6)$.
Weak adsorption of hydrogen onto the electrode surface made the reduction of benzoic acid possible, and the reduction was observed only at sp-metals of high hydrogen overvoltage. The specific adsorption of benzoic acid, as was observed on a lead electrode, also gave an important influence on the current efficiency for producing benzyl alcohol, i.e., the adsorption accelerated the rate of reduction of benzoic acid and the h.e.r. was partly inhibited by adsorbed benzoic acid molecules, thus the high current efficiency was obtained.

$$
<\text { E473> (Received Jul. 20, 1976) }
$$

\section{References :}

1) For example, "Organic Electrochemistry" ed. by $M$. M. Baizer (1973), Mercel Dekker, New York.

2) A.J. Appleby, "Modern Aspects of Electrochemistry," Vol. 9, p. 369 (1974), Plenum Pr., New York.

3) S. Ono, This Journal 23, 117 (1955); Bull. Chem. Soc. Japan 25, 404 (1952).

4) E.A. Efimov, I.G. Erusalimchik, Russ. J. Phys. Chem. 38, 1560 (1964).

5) J.A. Harrison, D.W. Shoesmith, J. Electroanal. Chem. 32, 125 (1971).

6) R.G. Barradas, O. Kutowy, D.W. Shoesmith, Electrochim. Acta 19, 49 (1974).

7) C. Mettler, Ber. 38, 1745 (1905).

8) F. Tupitsyn, N.K. Semenova, Tr. Gos. Inst. Prikl. Khim. 45, 25 (1960); C.A. 56, $1380 \mathrm{~g}$ (1962).

9) S. Swann, G.D. Luker, Trans, Electrochem. Soc. 75, 411 (1939).

10) J.W. Ross, R.D. Demars, I. Shain, Anal. Chem. 28, 1768 (1956).

11) I. Taniguchi, T. Sekine, This Journal 43, 709 (1975).

12) A.T. Petrenko, Russ. I. Phys. Chem. 39, 1119 (1965).

13) H. Kita, M. Honda, This Journal 38, 17 (1970).

14) B.B. Damaskin, O.A. Petrii, V.V. Batrakov, "Adsorption of Organic Compounds on Electrodes" p. 220 (1971), Plenum Pr., New York.

15) A. Aramata, P. Delahay, J. Phys. Chem. 68, 880 (1964).

16) L. Holleck, H. Marsen, Z. Elektrochem. 57, 301 (1953).

17) R.S. Perkins, T.N. Andersen, "Modern Aspects of Electrochemistry" Vol. 5, p. 252 (1969), Plenum Pr., New York.

18) L. Campanella, J. Electroanal. Chem. 28, 228 (1970).

19) A.P. Tomilov, S.G. Mairanovskii, M. Ya. Fioshin, V.A. Sminov, "Electrochemistry of Organic Compounds", p. 53 (1972), Halsted Pr., New York.

20) B. Kastening, L. Holleck, Z. Elektrochem. 63, 166 (1959).

21) H. Hosoya, S. Nagakura, Spectrochim. Acta 17, 324 (1961).

22) H. Kita, J. Electrochem. Soc. 113, 1095 (1966).

23) D.J.G. Ives, F.R. Smith, Trans, Faraday Soc. 63, 217 (1967). 